\section{Avifauna of wetlands of Amravati region, Maharashtra, India}

\section{Gajendrasingh Pachlore ${ }^{1}$ \& Mamata Chandrakar ${ }^{2}$}

${ }^{1}$ C/o Dr S.B. Pachlore, Sitaram Baba Colony, Ekvira Nagar, Pharshi Stop, Amravati, Maharashtra 444606, India

${ }^{2}$ C/o R.B. Chandrakar, Parvati apartment, Shegaon Naka, V.M.V. Road, Amravati, Maharashra 444604, India

Email: ${ }^{1}$ gpachlore@yahoo.com, ${ }^{2}$ mamatachandra@rediffmail.com

The avifauna of India and Pakistan was studied by Ali \& Ripley $(1987,1988)$. A total of 536 species were reported from India by Ali (2002). From the Amravati region, Wadatkar (2001) and Wadatkar \& Kasambe (2002) reported 171 species of birds from Pohra-Malkhed region and the Amravati University region.

This study is of immense importance to the wetlands which are mentioned here, as it has been observed that the water level of the wetlands is continuously declining and they are also getting polluted heavily by local human activities.

The source of water to all the lakes in this study is only rain water, thus the uncertainty in the amount of rainfall is another big reason for the shrinkage of a large area under water; eventually such poor and

Date of publication (online): 26 January 2011

Date of publication (print): 26 January 2011

ISSN $0974-7907$ (online) | 0974-7893 (print)

Editor: Rajah Jayapal

\section{Manuscript details:}

Ms \# 02034

Received 18 August 2008

Final received 12 November 2010

Finally accepted 25 November 2010

Citation: Pachlore, G. \& M. Chandrakar (2011). Avifauna of wetlands of Amravati region, Maharashtra, India Journal of Threatened Taxa 3(1) $1478-1484$

Copyright: (c) Gajendrasingh Pachlore \& Mamata Chandrakar 2011 Creative Commons Attribution 3.0 Unported License. JoTT allows unrestricted use of this article in any medium for non-profit purposes, reproduction and distribution by providing adequate credit to the authors and the source of publication.

Acknowledgements: The Authors are thankful to Dr. M.M. Baig, Head Department of Zoology, G.V.I.S.H Amravati for his help and support Authors are also thankful to colleagues Ananta, Pankaj Shelke, Parikshit Deshmukh, Gurav Lahe, Rakesh verma, Amit Mahalle. Authors would also like to extend thanks to Mr. Vikramsingh Pachlore, Director P.C.B.S.M.R.D.Institute, Amravati, and Mr. Pankaj Pachlore and Mrs Sarika Pachlore for providing valuable inputs in writing the manuscript.

\section{OPEN ACCESS | FREE DOWNLOAD (C) (i) (4)}

polluted wetlands are taken as the last choice by the migratory birds, hence causing a great loss to the richness of the wetlands.

A regular and meticulous study of these wetlands will definitely help to keep a record of birds species (resident and migratory), thus helping to restore as well as to maintain the present condition of all of the three wetlands.

\section{Study area}

Amravati is located at $20^{\circ} 93^{\prime \prime} \mathrm{N} \& 77^{0} 75^{\prime \prime} \mathrm{E}$, at an elevation of $343 \mathrm{~m}$ in Maharashtra. Three wetland areas of Amravati region have been studied which include Chhatri Lake, Wadali Lake and Bhivapur Lake or Talav.

Chhatri Lake $\left(20^{\circ} 53^{\prime} 42.6^{\prime \prime} \mathrm{N} \& 7^{\circ} 46^{\prime} 66.2\right.$ 'E, $372 \mathrm{~m}$ ) covers an area of $111.231934 \mathrm{~m}^{2}$ (Image 1).

Wadali Lake $\left(20^{\circ} 55^{\prime} 24.37^{\prime} \mathrm{N} \& 7^{0} 47^{\prime} 46.12^{\prime \prime} \mathrm{E}\right.$, $377 \mathrm{~m}$ ) covers an area of $77.818996 \mathrm{~m}^{2}$ (Image 2).

Bhivapur Lake (20055'79.1'N \& 77059'68.7'E, $352 \mathrm{~m}$ ) the largest of the three lakes is $162.744404 \mathrm{~m}^{2}$ in area; fishing is extensively done on a large commercial basis (Image 3 ).

Out of the three wetlands mentioned here, Chatri Talav and Wadali Talav are located in Amravati City and are thus taken care of by the Amravati Municipal Corporation (AMC); however, the AMC has given these two lakes on lease to other groups which are now responsible for the maintenance of the lakes and also earn profit by various activities like, boating, recreational games for children and snacks stalls for people of all ages who visit the lake.

Slightly different from the above two, the Bhivapur Talav is maintained by the Amravati Zillah Parishad (ZP). The ZP also takes out a tender of five years for any party interested in carrying out fishing activities at Bhivapur Talav, preference is always given to the fishing community.

The predominating vegetation is typically dry deciduous type (Champion \& Seth 1968). Common plant species are Acacia arabica, Azardirachta indica, Zizyphus jujuba, Eucalyptus sp., Lantana camara, Ipomoea fistula, Cassia sp. etc. Aquatic weeds of these areas are Hydrilla sp., Typha sp., Cyperus sp., Chara sp., among others.

The climate of Amravati is tropical wet and dry 


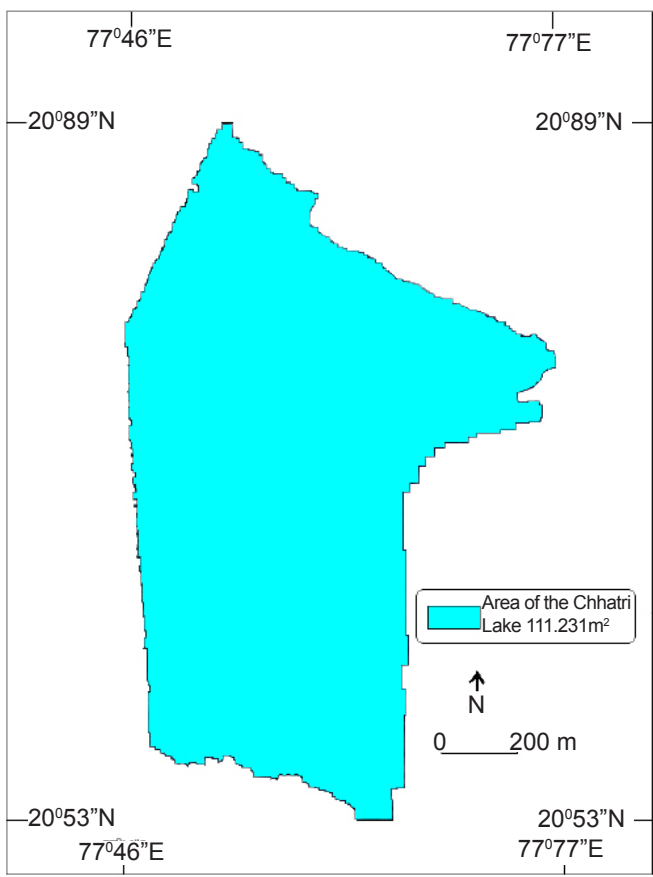

Image 1. Study area - Chhatri Lake
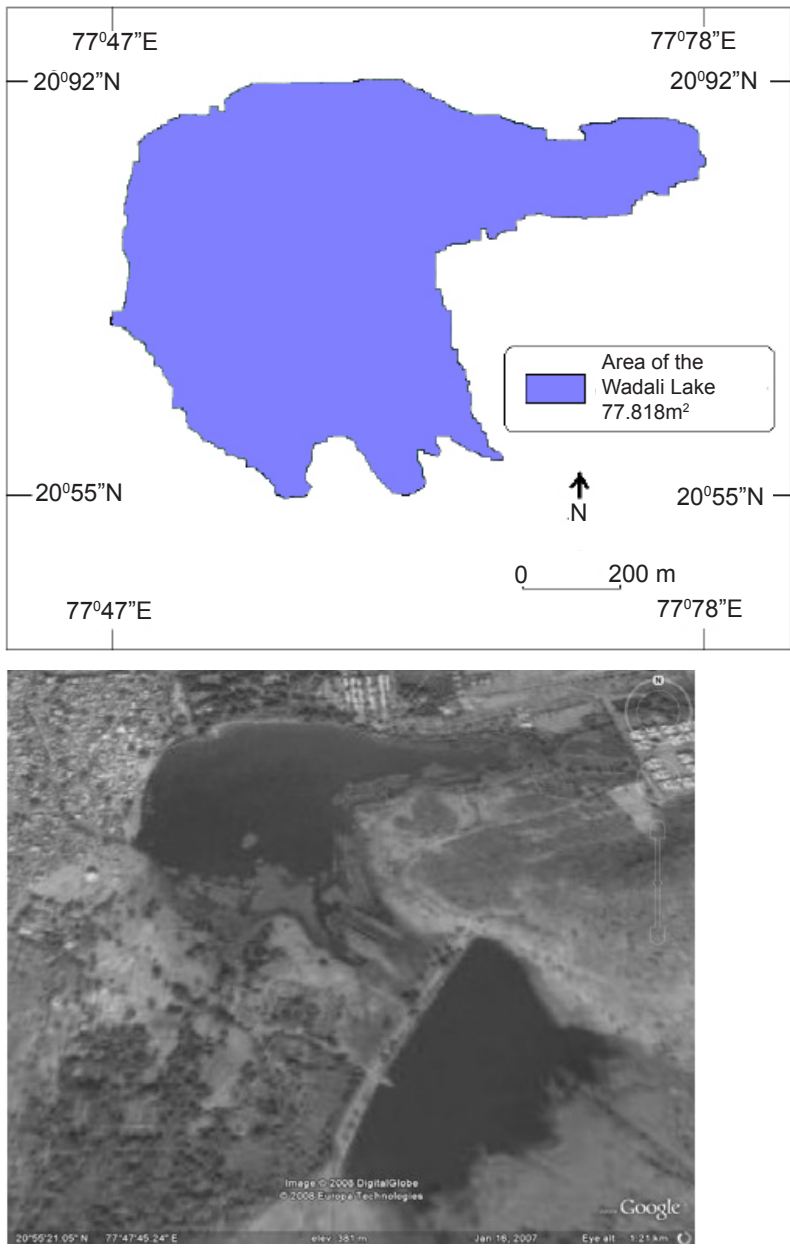

Image 2. Study area - Wadali Lake
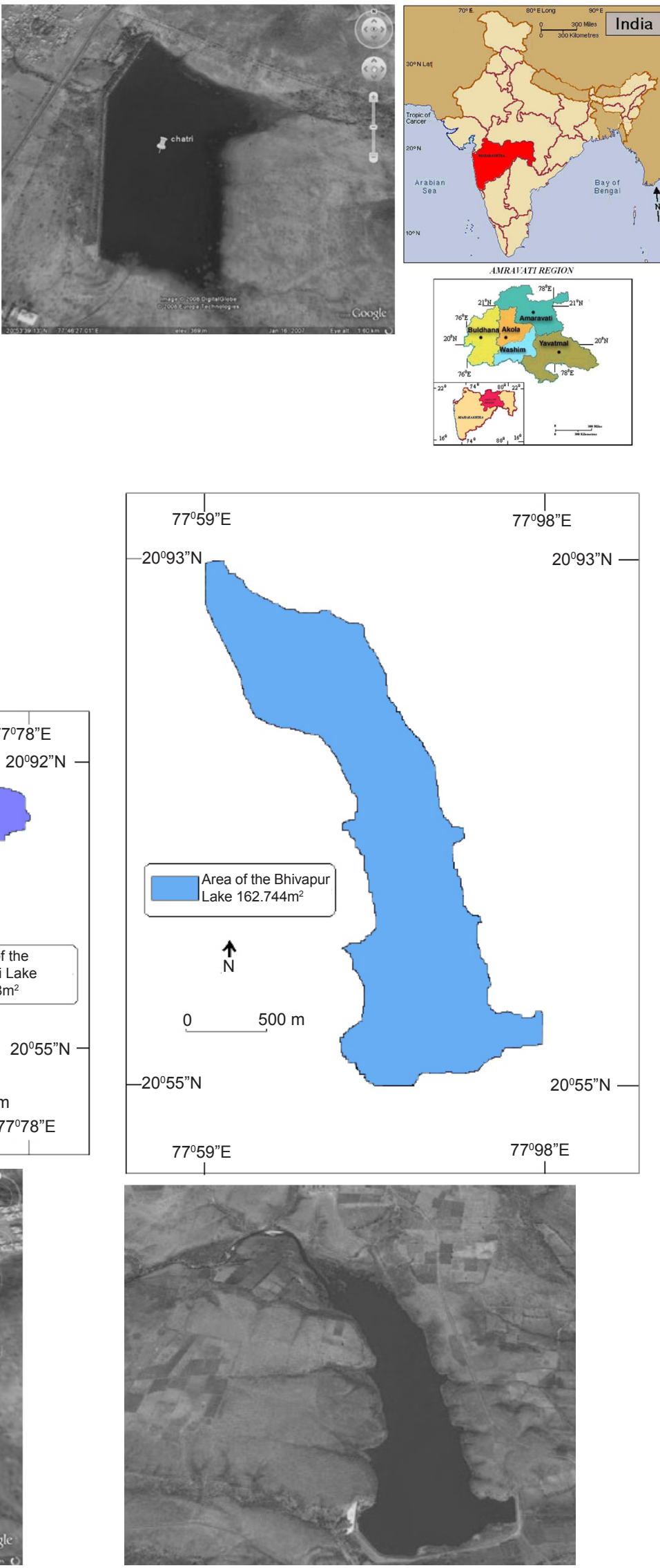

Image 3. Study area - Bhivapur Lake

Journal of Threatened Taxa I www.threatenedtaxa.org I January 2011 | 3(1): 1478-1484 
Table 1. Consolidated checklist of birds of wetlands of Amravati region, along with status, sighting months and sites of birds.

\begin{tabular}{|c|c|c|c|c|c|c|c|}
\hline & Common name & Scientific name & Status & $\begin{array}{l}\text { Month of } \\
\text { sight }\end{array}$ & \multicolumn{3}{|c|}{ Lake } \\
\hline & & & & & Chhatri & Wadali & Bhivapur \\
\hline & Podicipitidae & & & & & & \\
\hline \multirow[t]{2}{*}{1} & Little Grebe & Tachybaptus ruficollis & $\mathrm{R}$ & Mar & * & * & * \\
\hline & Phalacrocoracidae & & & & & & \\
\hline \multirow[t]{2}{*}{2} & Little Cormorant & Phalacrocorax niger & RM & Mar & * & * & * \\
\hline & Ardeidae & & & & & & \\
\hline 3 & Grey Heron & Ardea cinerea & $\mathrm{RM}$ & + & * & * & * \\
\hline 4 & Purple Heron & Ardea purpurea & RM & + & * & * & \\
\hline 5 & Black-crowned Night Heron & Nycticorax nycticorax & RM & + & * & * & * \\
\hline 6 & Large Egret & Casmerodius albus & RM & + & * & * & * \\
\hline 7 & Cattle Egret & Bubulcus ibis & $\mathrm{R}$ & + & * & * & * \\
\hline 8 & Little Egret & Egretta garzetta & $\mathrm{R}$ & + & * & * & * \\
\hline 9 & Yellow Bittern & Ixobrychus sinensis & $\mathrm{R}$ & + & * & * & \\
\hline \multirow[t]{2}{*}{10} & Great Bittern & Botaurus stellaris & M & Feb \# & & * & \\
\hline & Threskiornithidae & & & & & & \\
\hline 11 & Black Ibis & Pseudibis papillosa & RM & + & * & * & * \\
\hline 12 & Oriental White Ibis & Threskiornis melanocephalus & $\mathrm{R}$ & Mar & * & * & * \\
\hline \multirow[t]{2}{*}{13} & Glossy lbis & Plegadis falcinellus & RM & Jun & * & & \\
\hline & Ciconiidae & & & & & & \\
\hline 14 & White-necked Stork & Ciconia episcopus & $\mathrm{R}$ & Feb-Apr & * & * & * \\
\hline \multirow[t]{2}{*}{15} & Open-billed Stork & Anastomus oscitans & RM & Mar \# & & & * \\
\hline & Anatidae & & & & & & \\
\hline 16 & Brahminy Shelduck & Tadorna ferruginea & RM & Nov-Mar & * & * & * \\
\hline 17 & Northern Pintail & Anas acuta & M & Nov-Mar & * & * & * \\
\hline 18 & Common Teal & Anas crecca & M & Nov-Mar & * & & \\
\hline 19 & Spot-billed Duck & Anas poecilorhyncha & $\mathrm{RM}$ & + & * & * & * \\
\hline 20 & Eurasian Wigeon & Anas penelope & M & Nov-Feb & * & * & * \\
\hline 21 & Red-crested Pochard & Rhodonessa rufina & M & Nov-Feb & * & * & * \\
\hline 22 & Common Pochard & Aythya ferina & M & Nov-Feb & * & * & * \\
\hline 23 & Cotton Teal & Nettapus coromendelianus & $\mathrm{R}$ & + & * & * & * \\
\hline 24 & Garganey & Anas querquedula & M & Mar & * & * & * \\
\hline \multirow[t]{2}{*}{25} & Lesser Whistling Duck & Dendrocygna javanica & $\mathrm{R}$ & Mar & * & * & * \\
\hline & Accipitridae & & & & & & \\
\hline 26 & Black-shouldered Kite & Elanus caeruleus & $\mathrm{R}$ & + & * & * & * \\
\hline 27 & Black Kite & Milvus migrans & $\mathrm{R}$ & + & * & * & * \\
\hline 28 & Shikra & Accipiter badius & $\mathrm{R}$ & + & * & * & * \\
\hline \multirow[t]{2}{*}{29} & Western Marsh Harrier & Circus aeruginosus & M & Feb & & * & \\
\hline & Phasianidae & & & & & & \\
\hline 30 & Grey Francolin & Francolinus pondicerianus & $\mathrm{R}$ & + & * & * & \\
\hline \multirow[t]{2}{*}{31} & Indian Peafowl & Pavo cristatus & $\mathrm{R}$ & + & & * & \\
\hline & Rallidae & & & & & & \\
\hline 32 & Purple Moorhen & Porphyrio porphyrio & $\mathrm{R}$ & + & * & * & * \\
\hline 33 & Common Coot & Fulica atra & $\mathrm{R}$ & + & * & * & * \\
\hline 34 & White-breasted Waterhen & Amaurornis phoenicurus & $\mathrm{R}$ & + & * & * & * \\
\hline
\end{tabular}




\begin{tabular}{|c|c|c|c|c|c|c|c|}
\hline & \multirow[t]{2}{*}{ Common name } & \multirow[t]{2}{*}{ Scientific name } & \multirow[t]{2}{*}{ Status } & \multirow[t]{2}{*}{$\begin{array}{l}\text { Month of } \\
\text { sight }\end{array}$} & \multicolumn{3}{|c|}{ Lake } \\
\hline & & & & & Chhatri & Wadali & Bhivapur \\
\hline \multirow[t]{2}{*}{35} & Common Moorhen & Gallinula chloropus & RM & + & * & * & \\
\hline & Jacanidae & & & & & & \\
\hline 36 & Pheasant-tailed Jacana & Hydrophasianus chirurgus & $\mathrm{R}$ & Mar-Oct & * & * & \\
\hline \multirow[t]{2}{*}{37} & Bronze-winged Jacana & Metopidius indicus & $\mathrm{R}$ & Mar-Oct & * & * & \\
\hline & Charadriidae & & & & & & \\
\hline 38 & Yellow-wattled Lapwing & Vanellus indicus & $\mathrm{R}$ & + & * & * & * \\
\hline 39 & Red-wattled Lapwing & Vanellus malabaricus & $\mathrm{R}$ & + & * & * & * \\
\hline \multirow[t]{2}{*}{40} & Little Ringed Plover & Charadrius dubius & RM & + & * & * & * \\
\hline & Scolopacidae & & & & & & \\
\hline 41 & Common Sandpiper & Actitis hypoleucos & RM & June-mar & * & * & * \\
\hline \multirow[t]{2}{*}{42} & Wood Sandpiper & Tringa glareola & M & June-mar & * & * & * \\
\hline & Recurvirostridae & & & & & & \\
\hline \multirow[t]{2}{*}{43} & Black-winged Stilt & Himantopus himantopus & M & Nov- Mar & * & * & * \\
\hline & Burhinidae & & & & & & \\
\hline \multirow[t]{2}{*}{44} & Great Stone Plover & Esacus recurvirostris & $\mathrm{R}$ & \# & & & * \\
\hline & Glareolidae & & & & & & \\
\hline \multirow[t]{2}{*}{45} & Indian Courser & Cursorius coromandelicus & $\mathrm{R}$ & $\#$ & & & * \\
\hline & Rostratulidae & & & & & & \\
\hline 46 & Snipe sp. & Gallinago sp. & M & Dec-Feb. & * & * & \\
\hline \multirow[t]{2}{*}{47} & Greater Painted-snipe & Rostratula benghalensis & $\mathrm{R}$ & + & * & * & * \\
\hline & Laridae & & & & & & \\
\hline \multirow[t]{2}{*}{48} & River Tern & Sterna aurantia & RM & + & * & * & * \\
\hline & Columbidae & & & & & & \\
\hline 49 & Blue Rock Pigeon & Columba livia & $\mathrm{R}$ & + & * & * & * \\
\hline \multirow[t]{3}{*}{50} & Red Collared Dove & Streptopelia tranquebarica & $\mathrm{R}$ & + & * & * & * \\
\hline & Spotted Dove & Streptopeli chinensis & $\mathrm{R}$ & + & * & * & * \\
\hline & Pteroclididae & & & & & & \\
\hline \multirow[t]{2}{*}{51} & Painted Sandgrouse & Pterocles indicus & $\mathrm{R}$ & + & * & * & \\
\hline & Psittacidae & & & & & & \\
\hline \multirow[t]{2}{*}{52} & Rose-ringed Parakeet & Psittacula krameri & $\mathrm{R}$ & + & * & * & * \\
\hline & Cuculidae & & & & & & \\
\hline 53 & Greater Coucal & Centropus sinensis & $\mathrm{R}$ & + & * & * & * \\
\hline 54 & Pied Crested Cuckoo & Clamator jacobinus & $\mathrm{R}$ & May-Sept & & * & \\
\hline \multirow[t]{2}{*}{55} & Asian Koel & Eudynamys scolopacea & $\mathrm{R}$ & + & * & * & * \\
\hline & Tytonidae & & & & & & \\
\hline \multirow[t]{2}{*}{56} & Barn Owl & Tyto alba & $\mathrm{R}$ & + & * & * & * \\
\hline & Strigidae & & & & & & \\
\hline \multirow[t]{2}{*}{57} & Brown Fish Owl & Ketupa zeylonensis & $\mathrm{R}$ & $\#$ & * & & \\
\hline & Apodidae & & & & & & \\
\hline 58 & Asian Palm Swift & Cypsiurus balasiensis & $\mathrm{R}$ & + & * & * & * \\
\hline \multirow[t]{2}{*}{59} & House Swift & Apus affinis & $\mathrm{R}$ & + & * & * & * \\
\hline & Alcedinidae & & & & & & \\
\hline 60 & Small Blue Kingfisher & Alcedo atthis & RM & + & * & * & * \\
\hline 61 & Lesser Pied Kingfisher & Ceryle rudis & $\mathrm{R}$ & + & * & * & * \\
\hline
\end{tabular}




\begin{tabular}{|c|c|c|c|c|c|c|c|}
\hline & Common name & Scientific name & Status & Month of & \multicolumn{3}{|c|}{ Lake } \\
\hline & & & & & Chhatri & Wadali & Bhivapur \\
\hline \multirow[t]{2}{*}{62} & White-breasted Kingfisher & Halcyon smyrnensis & $\mathrm{R}$ & + & * & * & * \\
\hline & Meropidae & & & & & & \\
\hline \multirow[t]{2}{*}{63} & Small Green Bee-eater & Merops orientalis & $\mathrm{R}$ & + & * & * & * \\
\hline & Coraciidae & & & & & & \\
\hline \multirow[t]{2}{*}{64} & Indian Roller & Coracias benghalensis & $\mathrm{R}$ & + & * & * & * \\
\hline & Upupidae & & & & & & \\
\hline \multirow[t]{2}{*}{65} & Common Hoopoe & Upupa epops & $\mathrm{R}$ & + & * & * & * \\
\hline & Alaudidae & & & & & & \\
\hline 66 & Ashy-crowned Sparrow Lark & Eremopterix grisea & $\mathrm{R}$ & + & * & * & * \\
\hline \multirow[t]{2}{*}{67} & Common Crested Lark & Galerida cristata & $\mathrm{R}$ & + & * & * & * \\
\hline & Hirundinidae & & & & & & \\
\hline \multirow[t]{2}{*}{68} & Wire-tailed Swallow & Hirundo smithii & $\mathrm{RM}$ & Oct-Mar & * & * & * \\
\hline & Dicruridae & & & & & & \\
\hline \multirow[t]{2}{*}{69} & Black Drongo & Dicrurus macrocercus & $\mathrm{R}$ & + & * & * & * \\
\hline & Sturnidae & & & & & & \\
\hline 70 & Brahminy Starling & Sturnus pagodarum & $\mathrm{R}$ & & * & * & * \\
\hline 71 & Asian Pied Starling & Sturnus contra & $\mathrm{R}$ & + & * & * & * \\
\hline 72 & Common Myna & Acridotheres tristis & $\mathrm{R}$ & + & * & * & * \\
\hline 73 & Jungle Myna & Acridotheres fuscus & $\mathrm{R}$ & + & * & * & * \\
\hline \multirow[t]{2}{*}{74} & Rosy Starling & Sturnus roseus & M & Mar-May & & * & \\
\hline & Corvidae & & & & & & \\
\hline 75 & House Crow & Corvus splendens & $\mathrm{R}$ & + & * & * & * \\
\hline \multirow[t]{2}{*}{76} & Indian Treepie & Dendrocitta vagabunda & $\mathrm{R}$ & Mar \# & & * & \\
\hline & Pycnonotidae & & & & & & \\
\hline \multirow[t]{2}{*}{77} & Red-vented Bulbul & Pycnonotus cafer & $\mathrm{R}$ & + & * & * & * \\
\hline & Timaliidae & & & & & & \\
\hline 78 & Yellow-eyed Babbler & Chrysomma sinensis & $\mathrm{R}$ & + & * & * & \\
\hline 79 & Jungle Babbler & Turdoides striatus & $\mathrm{R}$ & + & * & * & * \\
\hline \multirow[t]{2}{*}{80} & Large Grey Babbler & Turdoides malcolmi & $\mathrm{R}$ & + & * & * & * \\
\hline & Motacilladae & & & & & & \\
\hline 81 & Yellow Wagtail & Motacilla flava & $\mathrm{RM}$ & Nov- Mar & * & * & * \\
\hline 82 & Forest Wagtail & Dendronanthus indicus & $\mathrm{R}$ & + & * & * & * \\
\hline 83 & White Wagtail & Motacilla alba & $\mathrm{R}$ & + & * & * & * \\
\hline 84 & Paddyfield Pipit & Anthus rufulus & $\mathrm{R}$ & Jul-Nov & * & * & * \\
\hline \multirow[t]{2}{*}{85} & Large Pied Wagtail & Motacilla maderaspatensis & $\mathrm{R}$ & + & * & * & * \\
\hline & Sylviidae & & & & & & \\
\hline \multirow[t]{2}{*}{86} & Ashy Prinia & Prinia socialis & $\mathrm{R}$ & + & * & * & * \\
\hline & Turdidae & & & & & & \\
\hline 87 & Indian Robin & Saxicoloides fulicata & $\mathrm{R}$ & + & * & * & * \\
\hline 88 & Oriental Magpie-robin & Copsychus saularis & $\mathrm{R}$ & + & * & * & * \\
\hline 89 & Pied Bush Chat & Saxicola caprata & $\mathrm{R}$ & & * & * & * \\
\hline 90 & Bluethroat & Luscinia svecica & RM & $\mathrm{Feb}^{\#}$ & * & & \\
\hline 91 & Common Stone Chat & Saxicola torquata & RM & Dec & & * & \\
\hline
\end{tabular}




\begin{tabular}{|c|c|c|c|c|c|c|c|}
\hline & Common name & Scientific name & Status & $\begin{array}{c}\text { Month of } \\
\text { sight }\end{array}$ & \multicolumn{3}{|c|}{ Lake } \\
\hline & & & & & Chhatri & Wadali & Bhivapur \\
\hline & Nectariniidae & & & & & & \\
\hline \multirow[t]{2}{*}{92} & Purple Sunbird & Nectarinia asiatica & $\mathrm{R}$ & + & * & * & * \\
\hline & Daniidae & & & & & & \\
\hline 93 & Bay-backed Shrike & Lanius vittatus & $\mathrm{R}$ & + & * & * & * \\
\hline \multirow[t]{2}{*}{94} & Rufous-backed Shrike & Lanius schach & $\mathrm{R}$ & + & * & * & * \\
\hline & Muscicapidae & & & & & & \\
\hline \multirow[t]{2}{*}{95} & Asian Paradise-flycatcher & Terpsiphone paradise & RM & Mar \# & & * & \\
\hline & Ploceidae & & & & & & \\
\hline 96 & House Sparrow & Passer domesticus & $\mathrm{R}$ & + & * & * & * \\
\hline 97 & Baya Weaver & Ploceus phillippinus & $\mathrm{R}$ & June-Aug & * & * & * \\
\hline
\end{tabular}

The status (seasonal occurrence), feeding habits, months of bird sighting and place of occurrence in the various habitats have been worked out. Different parameters were used as below:

R - Resident; RM - Resident Migratory; M - Migratory; + - throughout the Year, \# - sighted once; * - Presence

climate with hot dry summers from March to June. The monsoon season is from July to October and warm winters from November to March; the highest and lowest temperature ever recorded was $46.7^{\circ} \mathrm{C}$ on 25 May 1954 and $5.0^{\circ} \mathrm{C}$ on 09 February 1887 respectively.

The Avifauna of these wetland areas has not yet been reported. This study will provide a base for further study.

\section{Methods}

The study is based on the observations of two years from March 2006 to March 2008. Regular visits were made to these study areas. In all 230 visits were made to each of the wetlands by three teams, comprising of minimum of three and maximum of 10 persons in each team. Observations were carried on a fixed path in a $1 \mathrm{~km}$ radius at each station by using the line transect method by Gaston (1973). The birds were observed during the peak hours of their activity from $0600 \mathrm{hr}$ to $1000 \mathrm{hr}$ and in the evening from $1600 \mathrm{hr}$ to $1800 \mathrm{hr}$. Observations were also made during other times of the day as per convenience. Classification followed in this study is as per Ali (2002).

\section{Observation}

Ninety-seven species of birds were recorded from three wetland areas of Amravati region (Table 1). Out of 97 species reported, 66 species were local or resident, 20 were resident migrant and 12 species were migrant. Wadali Lake region was found to be inhabited by the highest number of species i.e. 90 . This was followed by Chhatri Lake and Bhivapur Lake with species found to be 88 and 77 respectively . The eating habits revealed that the highest number of birds recorded were insectivores (48), followed by piscivores (21), omnivores (12), grainivores (11), carnivores (6), frugivores (3), nectarivores (1). Out of 97 taxa recorded, the Oriental White Ibis Threskiornis melanocephalus is listed as Near Threatened (IUCN 2007) globally and the Indian Peafowl Pavo cristatus is included in Schedule I of the Wildlife Protection Act 1972 (Arora 2003).

\section{Discussion}

During the period of entire survey, the pair of Black-headed Gull Larus ribdibundus was sighted only once at Chatri Talav during the winter of 2007. Also the Purple Swamp Hen Porphyrio porphyrio population shows seasonal fluctuation in the number of individuals. Although no bird count was done, it was observed during the field visits that the number of Purple Swamp Hen individuals were sometime found in large numbers during winter and rainy seasons, but remarkably low during summers.

Many of these wetlands are used for Ganesh and Durga idol immersion during the festival time of Ganesh Chaturti and Navratri. The total absence of management at the time of idol immersion pollutes these wetlands. Polluted shores of such wetlands have caused increases in the number of scavenger birds like the House Crow Corvus splendens which are 
found to be feeding on the eggs of Common Coots, Purple Moorhen, and Jacanas and are thus responsible for the decrease in their population. To maintain the biodiversity richness of these areas serious attempts should be made from the concerned authorities, as well as from the local population. Educating the local population and making them aware of these facts will increase the wetland biodiversity richness

\section{REFERENCES}

Ali, S. \& D.S. Ripley (1987). Compact Handbook of Birds of India \& Pakistan - $2^{\text {nd }}$ Edition. Oxford University Press, $737 \mathrm{pp}$.

Ali, S. (2002). The Book of Indian Birds. 13th revised edition. Bombay Natural History Society, Bombay, 326pp.

Ali, S. \& S.D. Ripley (1988). Compact Handbook of the Birds of India and Pakistan: together with those of Bangladesh, Nepal, Bhutan, and Sri Lanka. 2nd edition. Oxford University Press, 890pp.
Arora, K. (2003). Forest laws. The Wild Life Protection Act, 1972 as amended by the Wild (Protection) Amendment Act, 2002. Professional Book Publishers, New Delhi, 85pp.

Champion, H.G. \& S.K. Seth (1968). A Revised Survey of The Forest Types of India. Govt. of India, Delhi, 185pp.

Gaston, A.J. (1973). Methods for estimating bird population. Journal of the Bombay Natural History Society 72(2): 272281.

Grimmette, K., C. Inskipp \& T. Inskipp (1999). Birds of Indian Subcontinent . Oxford University Press, New Delhi, $384 \mathrm{pp}$.

IUCN (2010). IUCN Red List of Threatened Species. Version 2010.4. <www.iucnredlist.org>. Downloaded on 09 December 2010.

Wadatkar, J.S. (2001). Checklist of birds from Amravati University Campus, Amravati. Zoos' Print Journal 16(5): 497-499.

Wadatkar, J.S. \& R. Kasambe (2002). Checklist of Birds from Pohra-Malkhed Reserve Forest, District Amravti, Maharashtra. Zoos' Print Journal 17(6): 807-811.

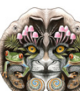

